\title{
3D visualization of the internal nanostructure of polyamide thin films in RO membranes
}

Federico Pacheco ${ }^{\mathrm{a}, \mathrm{b}^{*}}$, Rachid Sougrat ${ }^{\mathrm{c}}$, Martin Reinhard ${ }^{\mathrm{b}}$, James O. Leckie ${ }^{\mathrm{b}}$ and Ingo Pinnau ${ }^{\mathrm{a}^{*}}$

${ }^{a}$ Advanced Membranes \& Porous Materials Center, Division of Physical Sciences and Engineering, King Abdullah University of Science and Technology (KAUST), Thuwal 23955-6900, Kingdom of Saudi Arabia

${ }^{b}$ Department of Civil and Environmental Engineering, Stanford University, Jerry Yang \& Akiko Yamazaki Environment \& Energy Building, 473 Via Ortega, Stanford, CA 94305-4020, United States

${ }^{c}$ Imaging and Characterization Laboratory, King Abdullah University of Science and Technology (KAUST), Thuwal 23955-6900, Kingdom of Saudi Arabia

* Corresponding authors: phone: +96612808 2406

E-mail addresses: federico.pacheco@kaust.edu.sa (F. Pacheco) ingo.pinnau@ kaust.edu.sa (I. Pinnau)

Keywords: TEM, tomography, polyamide thin film, polyamide nanostructure, voids, 


\begin{abstract}
The front and back surfaces of fully aromatic polyamide thin films isolated from reverse osmosis (RO) membranes were characterized by TEM, SEM and AFM. The front surfaces were relatively rough showing polyamide protuberances of different sizes and shapes; the back surfaces were all consistently smoother with very similar granular textures formed by polyamide nodules of $20-50 \mathrm{~nm}$. Occasional pore openings of approximately the same size as the nodules were observed on the back surfaces. Because traditional microscopic imaging techniques provide limited information about the internal morphology of the thin films, TEM tomography was used to create detailed 3D visualizations that allowed the examination of any section of the thin film volume. These tomograms confirmed the existence of numerous voids within the thin films and revealed structural characteristics that support the water permeance difference between brackish water (BWRO) and seawater (SWRO) RO membranes. Consistent with a higher water permeance, the thin film of the BWRO membrane ESPA3 contained relatively more voids and thinner sections of polyamide than the SWRO membrane SWC3. According to the tomograms, most voids originate near the back surface and many extend all the way to the front surface shaping the polyamide protuberances. Although it is possible for the internal voids to be connected to the outside through the pore openings on the back surface, it was verified that some of these voids comprise nanobubbles that are completely encapsulated by polyamide. TEM tomography is a powerful technique for investigating the internal nanostructure of polyamide thin films. A comprehensive knowledge of the nanostructural distribution of voids and polyamide sections within the thin film may lead to a better understanding of mass transport and rejection mechanisms in RO membranes.
\end{abstract}




\section{Introduction}

The permeation and separation properties of thin film composite (TFC) reverse osmosis (RO) membranes are dictated by the structural and physicochemical characteristics of their surface polyamide thin films. These films are formed by interfacial polymerization (IP) of monomeric diamines dissolved in an aqueous solution — applied directly on a porous polysulfone support—and monomeric acid chlorides dissolved in a water-immiscible organic phase that is brought into contact with the aqueous solution [1-4]. Typical monomers used in the IP are $m$-phenylenediamine (MPD) and trimesoyl chloride (TMC), which create a cross-linked, fully aromatic polyamide network, and result in RO membranes with rough surfaces commonly referred to as ridge and valley structures [3]. Characterization of polyamide thin films is challenging due to their complex and irregular morphology and average thicknesses below $250 \mathrm{~nm}$ [5]. Consequently, a complete understanding of how the physical nanostructure of polyamide thin films ultimately determines membrane transport and rejection mechanisms has yet to be established.

According to the pioneering work by Morgan and Kwolek [4], polyamide IP occurs in the organic side of the interface due to the very low aqueous solubility of acid chlorides. Because of this imbalance in monomer solubility, polyamide formation depends on the partition and diffusion of the diamines into the organic solvent. The resulting thin film tends to grow perpendicular to and away from the interface until it becomes a physical barrier to further diffusion of diamine monomer, thus slowing and eventually terminating the IP. This process generates a morphological asymmetry in the thin film: the surface facing the aqueous phase is always smoother than the surface facing the organic phase [4]. It is important to clarify that this is only a relative comparison between the two surfaces of a given polyamide thin film, and does not necessarily mean that the organic side surface must be appreciably rough. Although these results were based primarily on the study of the linear aliphatic 6-10 polyamide system (i.e., the IP product of 1,6-diaminohexane and sebacoyl chloride), they extend to aromatic polyamide thin films as confirmed by John Cadotte during the creation of the FT-30 membrane, the first commercial TFC RO membrane based on the MPD-TMC chemistry [1-3].

As reported by Petersen and Cadotte [3], the distinctive morphological feature of the MPD-TMC polyamide thin film is its relatively rough front surface (i.e., the surface formed facing the organic side, 
and thus the surface of the TFC membrane), which they described as having "a well-developed 'ridge and valley' structure" [3] with "protuberances coming out of the plane of the membrane" [2]. This pronounced roughness was thought to be intrinsically associated with the use of aromatic diamine monomers, as other polyamide thin films made by the IP of monomeric and polymeric aliphatic diamines produce thin films with considerably flatter front surfaces [3,6]. Departing from the IP strategy, Johnson et al. demonstrated that flat MPD-TMC polyamide thin films can be created through a molecular layer-by-layer deposition process [7]. Recently, Karan and co-workers were able to fabricate extremely smooth and thin $(<10 \mathrm{~nm})$ MPD-TMC polyamide films through an ingenious IP method using very low MPD concentrations $(\leq 0.1 \mathrm{wt} \%)$ [8]. However, they did not report testing membranes with these remarkable thin films under RO conditions. When thin films were made with MPD concentrations similar to those conventionally used for making RO membranes (i.e., $2 \mathrm{wt} \%$ ) [3], the rough ridge and valley front surface appeared once again [8].

Petersen and Cadotte also examined the back surface of the thin film (i.e., the surface formed facing the aqueous side and the polysulfone support) and described it as possessing "numerous micropores or passageways for exit of permeate water from the depths of the interfacial membrane" [3]. The origin of these pores was attributed to the IP mechanism, in which a porous incipient film is created allowing further diffusion of diamine until all openings are sealed during the subsequent growth of the film [2]. Since the development of the FT-30 membrane, limited characterization work has been done on the morphology of back surfaces of MPD-TMC-based polyamide thin films [8-13]. Nevertheless, the presence of pores was confirmed consistently regardless of whether the thin films came from RO membranes (i.e., formed directly on porous supports) or whether they were made as stand-alone films. Yan and co-workers reported comparable back surface average pore sizes around $20 \mathrm{~nm}$ for three different membranes [13].

Today, most commercial TFC RO membranes - including those with additional coatings - have polyamide thin films based on the IP of MPD and TMC [14-16]. It is quite noteworthy that membranes containing thin films made of these monomers can show such versatility in performance, spanning a wide range of applications from nanofiltration (NF) to seawater desalination, as well as non-aqueous liquid separations [17]. This is possible because of the complexity of the IP process, which is influenced 
by many variables including monomer concentrations, choice of solvents, additives, porous support properties and curing time. All these parameters affect the mass transfer of diamine to the organic phase and therefore the formation and resulting physicochemical characteristics of the thin film [1-4]. Customarily, changes in IP conditions are evaluated based on water permeance and salt rejection of the final membranes. Optimizing IP process conditions to tailor membrane performance has been the focus of extensive research spanning four decades.

We are particularly interested in the subset of studies that have documented how variables of the MPDTMC IP process affect the nanostructure and physical morphology of polyamide thin films. Replacing MPD with its isomer $p$-phenylenediamine (PPD) resulted in a membrane with a rough surface that contained, however, more regularly packed globules [6]. Ghosh et al. [18] altered the partition coefficient and diffusivity of MPD by using several organic solvents. Although membrane performance was affected, no significant surface morphology difference was detected from SEM images. Hirose and co-workers [19] added up to $20 \mathrm{wt} \%$ isopropyl alcohol (IPA) to the aqueous phase and recorded improvements in water flux (without affecting salt rejection). In addition, surface roughness increased with higher IPA concentrations. Above $20 \mathrm{wt} \%$ IPA, membrane performance deteriorated rapidly and surface morphology shifted from the ridge and valley to a crater-like structure [19]. Another study obtained similar results when mixing dimethyl sulfoxide (DMSO) with the aqueous phase [20]. The increase in roughness was attributed to fluctuations in the water-hexane interface due to enhanced solvent miscibility provided by the DMSO, which also facilitated the diffusion of MPD. Following a different approach, Kong et al. [21] used acetone as a co-solvent of hexane in the organic phase and produced a membrane with a relatively flat front surface. Closer inspection by TEM revealed a complex, multi-level internal structure with very thin sections $(8 \mathrm{~nm})$ of dense polyamide. Researchers from the same group [22] explored further the use of co-solvents in the organic phase and observed similar trends with diethyl ether and ethyl acetate. Surface roughness and the appearance of multi-level structures increased up to a certain co-solvent concentration, after which membranes became flatter and performance worsened. The effects of other additives that work as surfactants, catalysts or acid acceptors, such as triethylamine (TEA) and camphor sulfonic acid (CSA), have also been evaluated [18,23-25]. Furthermore, physicochemical properties of the porous support such as porosity and hydrophobicity play a role in shaping the morphology of the polyamide thin film by influencing the way in which the MPD aqueous solution impregnates the support [8,26-28]. This has been shown 
exemplarily by Karan and co-workers [8], who used a sacrificial support with high porosity, but very small pores, to control the delivery of MPD in the IP process and achieve both smooth and rough MPDTMC polyamide thin films.

Microscopic techniques based on atomic force microscopy (AFM), scanning electron microscopy (SEM) and transmission electron microscopy (TEM) are the predominant characterization tools for inspecting the physical morphology of polyamide thin films at nanometer scales [5,6,8,13,16,19,22,23,27,29-33]. Prompted in part by TEM images of the multi-level thin films obtained with organic co-solvents [21, 34], recent research has examined the internal structure of MPD-TMC polyamide thin films more closely, showing the presence of significant voids inside the major polyamide ridges or protuberances as well as significantly thinner sections of dense polymer [13,22,35,36].

In previous work [32], we introduced a TEM technique that produced 2D projections of isolated polyamide thin films and expanded the characterization applications of TEM beyond cross-section images $[8,13,37,38]$. Because these images contain structural information of the entire thin film volume, it is therefore possible to employ TEM tomography to obtain 3D visualizations of the thin films. The technique involves acquiring a series of $2 \mathrm{D}$ projection images at different tilt angles, which are then aligned and assembled in a stack, thus constructing the 3D image. The use of TEM tomography to characterize TFC membranes has seldom been reported [36,39,40]. The only detailed study is by She et al. [39], who essentially looked at a section of the porous support of a TFC NF membrane to demonstrate the advantages of the technique. Kurihara and Hanakawa [36] presented a 3D reconstruction of a sole protuberance of a polyamide thin film to prove its hollow core, but did not provide any experimental details on the technique or any images of the thin film. Departing from the TFC membranes, Nunes et al. [40] used TEM tomography to visualize the structure of micelles in an isoporous film. Here, we used TEM tomography to generate 3D images of two MPD-TMC polyamide thin films. These tomograms allowed us to reach and closely examine any location of the polyamide thin film volume revealing the distribution of polyamide and confirming the existence of numerous voids in the internal nanostructure. This work highlights TEM tomography as a characterization tool with the potential to significantly contribute in deciphering the complex morphology of polyamide thin films, which is one of the crucial steps towards better understanding mass transport, rejection and even fouling mechanisms in TFC RO membranes. 


\section{Materials and methods}

\subsection{Chemicals and membranes}

LR White resin and ACS grade chloroform were obtained from Polysciences (Warrington, PA) and Mallinckrodt Baker (Phillipsburg, NJ), respectively. Three commercial fully aromatic polyamide TFC RO membranes were studied: brackish water (BWRO) membranes ESPA3 from Hydranautics (Oceanside, CA) and AK from GE (Minnetonka, MN), and seawater (SWRO) membrane SWC3 from Hydranautics. All membranes have a polysulfone support and are backed by non-woven polyester fabrics for mechanical stability. The surfaces of the polysulfone supports of the BWRO membranes were also characterized. Polysulfone supports equivalent to those of ESPA3 and AK but without the polyamide thin films were obtained from the manufacturers.

\subsection{Isolating polyamide thin films for microscopic analysis}

In order to study the polyamide nanostructure it was necessary to isolate the thin films from the support membrane layers. Isolated polyamide thin films were collected on substrates suitable for the different microscopy methods (e.g., a copper grid for TEM or a $5 \mathrm{~mm}$ x $7 \mathrm{~mm}$ silicon chip for AFM) using a simple apparatus and procedure described in [32]. Briefly, membrane samples without the polyester backing were placed (surface facing down) onto the substrate, which was held in place in the apparatus by flat-tip reverse tweezers. The polysulfone was then dissolved and washed away with a slow stream of chloroform delivered from a burette positioned above the sample. This method produced samples of the back surface of the polyamide thin film. By placing the membrane piece onto the substrate with the surface facing up, specimens of the rough front surface of isolated polyamide thin films were obtained. These samples were significantly more difficult to prepare because the extremely fragile polyamide thin film was easily flushed away by the solvent. Loss of the thin film was often prevented by also holding a corner of the membrane piece with the tweezers.

\subsection{Transmission Electron Microscopy (TEM)}

\subsubsection{Cross-section and $2 \mathrm{D}$ projection images}

Images were acquired with a Jeol $1230 \mathrm{TEM}$ at an accelerating voltage of $80 \mathrm{kV}$ using Gatan CCD cameras (Pleasanton, CA), either a cooled 967 slow-scan or a high-resolution Orius SC1000A. Polyamide film features were measured directly from the images using the scale bars and the ruler tool 
in Adobe Photoshop ${ }^{\circledR}$. To obtain regular TEM cross-sections, membrane samples without the polyester backing were embedded in LR White resin and mounted onto carbon/Formvar ${ }^{\circledR}$ (polyvinyl formal) coated copper grids following a previously described procedure [32]. Cross-sections of isolated polyamide thin films were prepared by dipping grids holding regular TEM cross-sections three times in chloroform for $45 \mathrm{~s}$ each to remove the polysulfone support from the samples. It is important to mention that the copper grids were first exposed to the TEM beam before the cross-sections were mounted. This preliminary step was essential to protect the Formvar ${ }^{\circledR}$ layer of the grid from dissolving and consequently destroying the sample during the treatment with chloroform. The isolated polyamide thin film samples of ESPA3 contained $30 \mathrm{~nm}$ gold nanoparticles in the ridge and valley structure, which were used as reference markers during the development of the technique [32]. Samples for 2D projection images were prepared from isolated polyamide thin films following the procedure reported in the same previous study [32].

\subsubsection{TEM Tomography}

Tomograms were obtained from isolated polyamide thin films placed on 200-mesh uncoated copper TEM grids. Before imaging, the grids were floated on a drop of $15 \mathrm{~nm}$ gold nanoparticle suspension (Protein A, BBI Solutions, Cardiff, UK). Gold nanoparticles deposited on the polyamide thin films served as fiducial markers during alignment and reconstruction of the image series. Samples were imaged with an FEI Titan TEM (Eindhoven, Netherlands) operated at $300 \mathrm{kV}$ and equipped with a Gatan CCD camera. A series of images at different tilt angles ranging from $-70^{\circ}$ to $+70^{\circ}$ was acquired using FEI Xplore $3 \mathrm{D}$ tomography software starting at $0^{\circ}$ and following a Saxton tilting scheme with $2^{\circ}$ initial increments [41]. Almost 100 images were obtained for each tilt series, which were then aligned and assembled using a back-projection algorithm with the open-source software IMOD. Images and video animations of the 3D reconstructions were prepared using the tools available in IMOD and the Avizo ${ }^{\circledR}$ software package.

\subsection{Scanning electron microscopy (SEM)}

SEM micrographs of the front and back surfaces of thin films were obtained with FEI XL30 Sirion and Nova Nano630 microscopes at accelerating voltages of up to $5 \mathrm{kV}$. Samples were sputter-coated with a uniform layer of either palladium/gold or iridium to prevent charging effects. 


\subsection{Atomic force microscopy (AFM)}

AFM measurements were performed with a NanoScope IV MultiMode scanning probe microscope (Veeco Metrology Inc., Santa Barbara, CA) equipped with a J type scanner. Images were acquired in tapping mode from dry membrane samples at room temperature using RTESP etched silicon probes (Veeco Probes, Santa Barbara, CA). Before calculating roughness values, each image was individually inspected and, when needed, a second or third order polynomial was applied to flatten the image and correct for scanning artifacts (NanoScope software version 5.12). Cross-sectional profiles were prepared with the software program Gwyddion.

\section{Results and discussion}

\subsection{The front surface of fully aromatic polyamide thin films}

Fig. 1a and 1b present surface SEM micrographs of the BWRO membranes ESPA3 and AK, respectively. Made by different manufacturers, both membranes consist of fully aromatic polyamide formed from MPD and TMC, but their ridge and valley structures differ. Compared to ESPA3, which shows a rougher surface with more polyamide ridges blocking access to the thin regions (valleys), the surface of $\mathrm{AK}$ is relatively open with fewer and smaller ridges. In addition, globular polyamide features are observed in the valleys of the AK front surface. Examples in the literature show that incorporating different types of additives that promote mixing of the aqueous-organic interface during IP can produce polyamide thin films whose front-surface morphologies shift from a more regular structure (similar to that of AK) to a rougher, more pronounced ridge and valley structure (like that of ESPA3) while maintaining comparable performance [19,22,23]. Using the manufacturers' datasheets, we calculated water permeance values of $6.5 \mathrm{~L} \mathrm{~m}^{-2} \mathrm{~h}^{-1} \mathrm{bar}^{-1}$ and $5.9 \mathrm{~L} \mathrm{~m}^{-2} \mathrm{~h}^{-1} \mathrm{bar}^{-1}$ for ESPA3 and AK, respectively, corroborating comparable performances despite their front surface differences.

Cross-section images of the isolated polyamide thin films of ESPA3 and AK are shown in Fig. 1c and 1d, respectively. An unobstructed visualization of the nanostructure of these thin films is achieved by removing the polysulfone support. In conventional TEM cross-section images, the true extent of polyamide thin films is difficult to establish because the interface with the polysulfone support is almost never discernible. Consistent with the SEM micrographs, the ridge and valley structure of ESPA3 (Fig. 1c) extends further outward from the polysulfone support than that of AK (Fig. 1d) increasing both its 
surface roughness and average thin film thickness. These cross-section images show that despite physical differences between their front surfaces, both polyamide thin films have essentially the same overall asymmetric nanostructure: (1) a base of closely-packed nodular polyamide at the bottom of the thin film that forms a relatively smooth interface with the polysulfone support, and (2) a rough ridge and valley structure that grows outward from this base. It was previously reported that the nodular base of ESPA3 had an approximate thickness of 30-60 nm [32]. Similar measurements from cross-section TEM images established that for AK the nodular base was slightly thinner $(15-40 \mathrm{~nm})$, which is evident when comparing Fig. 1c and 1d. 

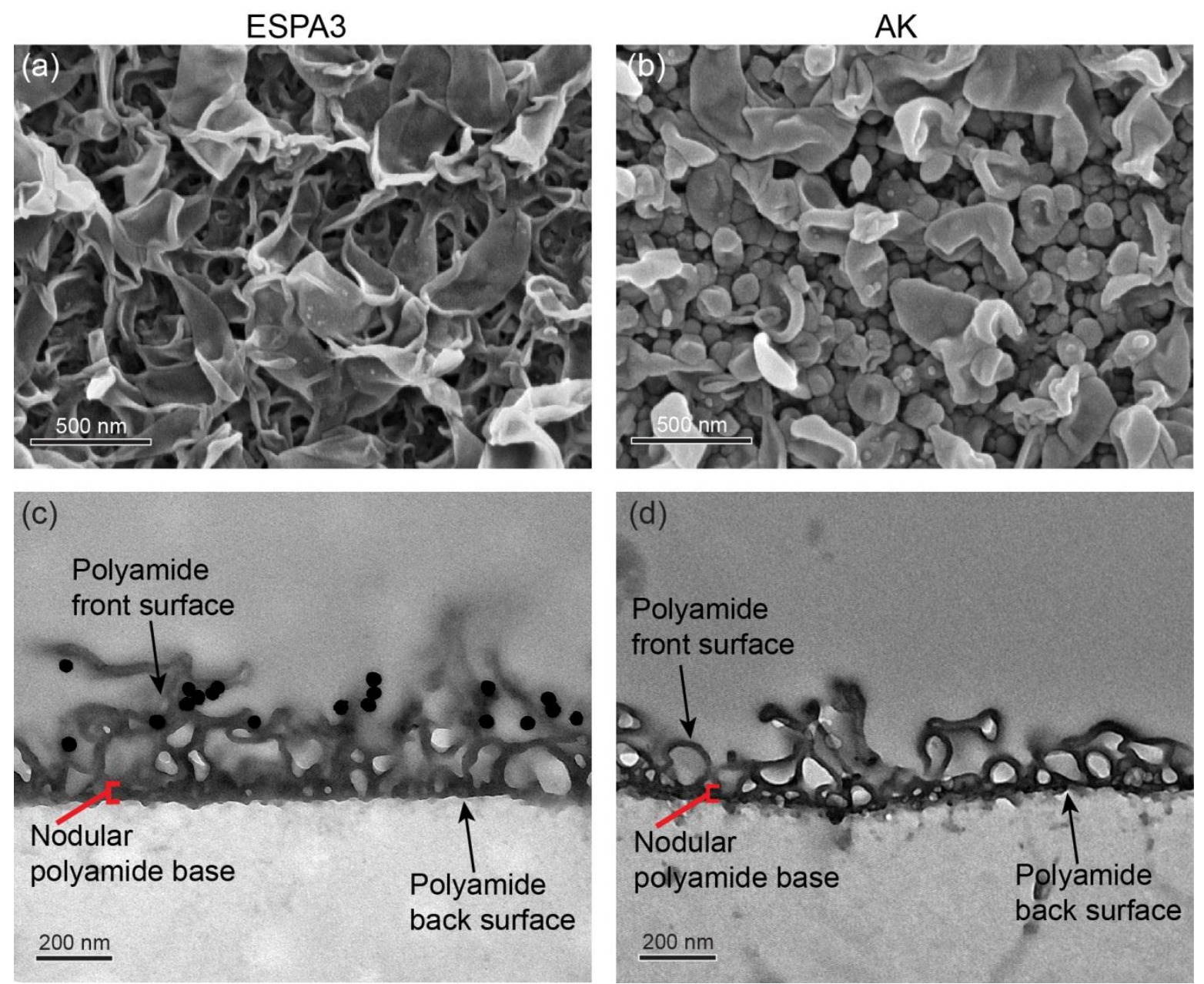

Fig. 1. Electron micrographs of the fully aromatic polyamide TFC BWRO membranes ESPA3 and AK: (a-b) surface SEM; (c-d) TEM cross-sections of the isolated thin films. The square brackets delimit the nodular polyamide base that forms the back surface of both thin films. The black dots in (c) are $30 \mathrm{~nm}$ gold nanoparticles [32]. Scale bars: (a-b) $500 \mathrm{~nm}$ (identical); (c-d) $200 \mathrm{~nm}$ (identical).

Table 1 summarizes AFM results for the two membranes. As described in Section 2.2, polyamide thin film back surfaces were uncovered by dissolving the polysulfone support with chloroform. Importantly, the $\mathrm{R}_{\mathrm{RMS}}$ values for both the front surface of regular ESPA3 samples and the front surface of isolated polyamide thin films of ESPA3 were virtually identical (135 nm and $132 \mathrm{~nm}$, respectively), indicating that thin film morphology was not changed by the dissolution of the polysulfone support. The same was concluded after inspecting and comparing SEM images of both pristine and isolated types (not shown). 
Furthermore, work by Bason et al. [42] proved that isolated polyamide thin films retained their reverse osmosis transport properties following polysulfone support removal with an organic solvent.

Table 1. Summary of AFM data of the front and back surfaces of the polyamide thin films of two commercial brackish water RO membranes. Reported are the averages and standard deviations.

\begin{tabular}{llcccc}
\hline Membrane & Thin Film Surface & Scans $^{\mathrm{a}}$ & $\begin{array}{c}\mathrm{R}_{\mathrm{RMS}}{ }^{\mathrm{b}} \\
{[\mathrm{nm}]}\end{array}$ & $\begin{array}{c}\mathrm{R}_{\mathrm{max}}^{\mathrm{c}} \\
{[\mathrm{nm}]}\end{array}$ & $\begin{array}{c}\text { Excess surface } \\
\text { area }^{\mathrm{d}}[\%]\end{array}$ \\
\hline ESPA3 & Front surface & 18 & $135 \pm 20$ & $906 \pm 138$ & $124 \pm 11$ \\
& Isolated front surface & 11 & $132 \pm 12$ & $859 \pm 100$ & $119 \pm 17$ \\
& Back surface & 17 & $20 \pm 9$ & $179 \pm 61$ & $18 \pm 5$ \\
& Front surface & 9 & $84 \pm 8$ & $571 \pm 67$ & $80 \pm 7$ \\
& Back surface & 5 & $19 \pm 5$ & $162 \pm 54$ & $24 \pm 1$ \\
\hline $\begin{array}{l}\text { Polysulfone } \\
\text { (Hydranautics) }\end{array}$ & Surface & 6 & $6.1 \pm 0.8$ & $93 \pm 65$ & $4 \pm 2$ \\
\hline $\begin{array}{l}\text { Polysulfone } \\
(\text { GE Osmonics) }\end{array}$ & Surface & & & & $7 \pm 2$ \\
\hline
\end{tabular}

${ }^{\mathrm{a}}$ Scan area $=5 \mu \mathrm{m} \times 5 \mu \mathrm{m}$.

${ }^{\mathrm{b}} \mathrm{R}_{\mathrm{RMS}}$ : root mean square roughness.

${ }^{c} R_{\text {max }}$ : maximum vertical distance between the highest and lowest points (i.e., maximum peak-to-valley vertical distance).

${ }^{\mathrm{d}}$ Percentage by which sample surface area exceeds the scan area $(5 \mu \mathrm{m} \times 5 \mu \mathrm{m})$.

${ }^{\mathrm{e}}$ Front surface of a polyamide thin isolated from the rest of the membrane with the chloroform technique.

Consistent with the analysis of Fig. 1, AFM measurements confirmed quantitatively the higher frontsurface roughness of ESPA3 compared to AK. Moreover, the $\mathrm{R}_{\max }$ values listed in Table 1 support the observation that the ridge and valley structure of ESPA3 extends further outward than that of AK, thus increasing its average thin film thickness. The asymmetric nanostructure of the thin films was also 
evidenced by the much higher $\mathrm{R}_{\mathrm{RMS}}$ values of the front surfaces compared to the back surfaces (Table 1 and Fig. S1 in the Supplementary Information).

\subsection{The back surface of fully aromatic polyamide thin films}

Fig. 2 offers a detailed view of the polyamide thin film back surfaces of the two membranes by combining SEM and high-resolution AFM images at identical scales. The back surfaces of ESPA3 (Fig. 2a) and AK (Fig. 2b) appear indistinguishable from one another with very similar granular textures, slight roughness, and moderate local height variability. These features seem to stem from polyamide nodules being fused together to form a continuous solid layer. Both back surfaces have nearly identical $\mathrm{R}_{\mathrm{RMS}}$ values of approximately $20 \mathrm{~nm}$ (Table 1), corroborating their similarity and relatively smooth structures. The $\mathrm{R}_{\mathrm{RMS}}$ values are close to those reported by Karan et al. [8].
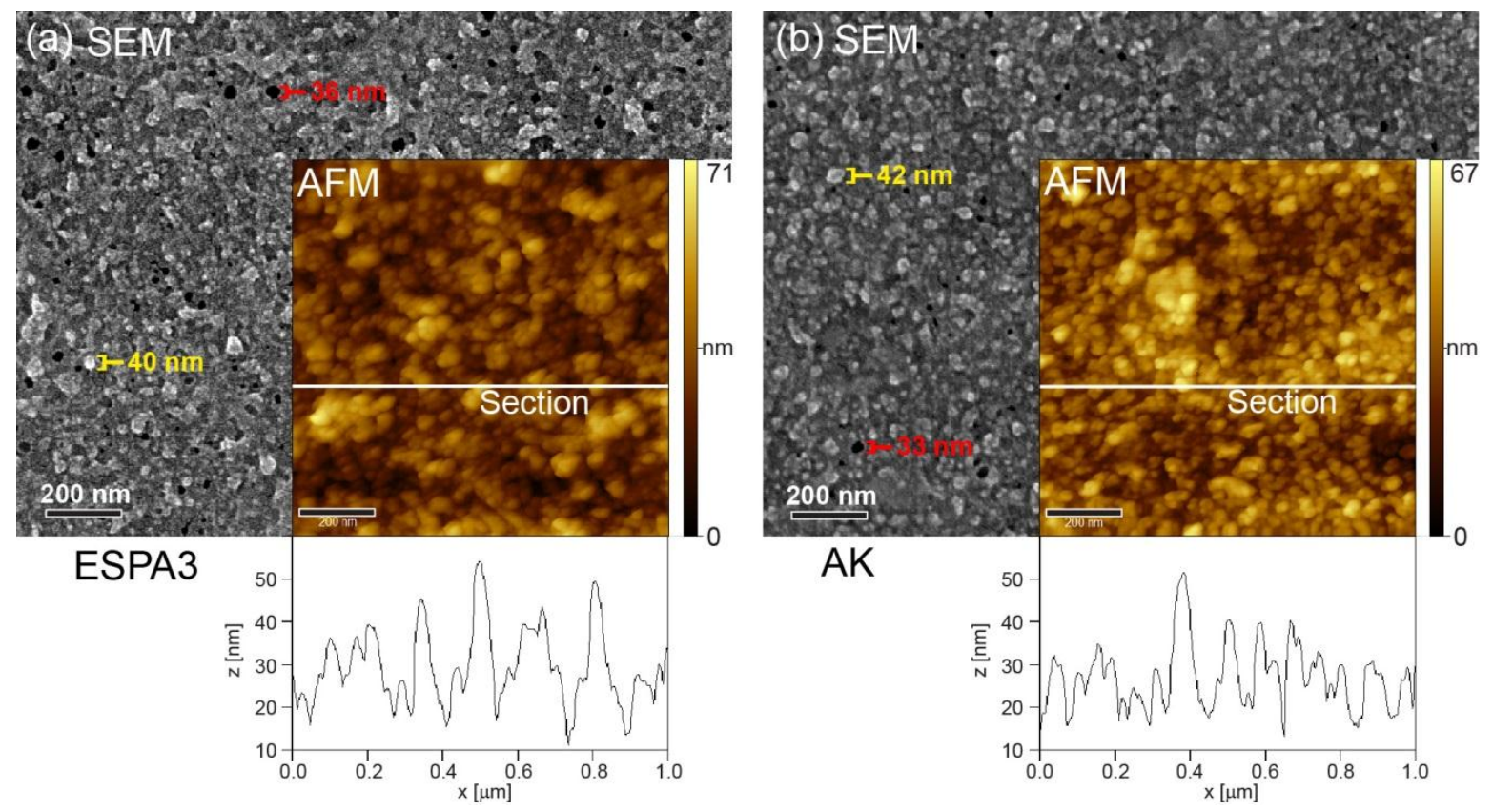

Fig. 2. SEM and AFM (1 $\mu \mathrm{m} \times 1 \mu \mathrm{m}$ scan area) images of the polyamide thin film back surface of (a) ESPA3 and (b) AK. Scale bars: $200 \mathrm{~nm}$ (identical for all images). The white lines on the AFM images mark the location at which the cross-sectional height profiles were obtained. Highlighted with yellow and red are conspicuous nodules and pore openings, respectively. The units of the $\mathrm{x}$ and $\mathrm{z}$ axes of the section profiles are $\mu \mathrm{m}$ and $\mathrm{nm}$, respectively. 
One particularly large nodule $(\sim 40 \mathrm{~nm})$ is marked with a yellow bracket in each image to illustrate their relative size. We determined from SEM images and AFM cross-sectional profiles, like those shown in Fig. 2, that nodule sizes ranged roughly from $20 \mathrm{~nm}$ to $50 \mathrm{~nm}$, with most of them being smaller than the ones selected in the images.

Previous studies of the back surface of polyamide thin films have highlighted its relative smoothness, but have not described nodules or any texture [8-13]. In our previous work [32], we referred to the bottom part of the thin film as a "nodular polyamide base" because of the presence of these small nodules. Although we did not show SEM images in [32], we were aware of the morphology of the back surface [33]. Inadvertently, by using the word "nodule" we may have contributed to the existing ambiguity that is inherent in how polyamide thin film features have been described in the literature. Other researchers $[6,18,28]$ have used the term "nodule" to define round, front-surface protuberances similar to those observed on the AK membrane (Fig. 1b). The cross-section of one of these round protuberances can be seen directly below the down-pointing arrow in Fig. 1d. Clearly, this is part of the ridge and valley structure and not of the base of the polyamide thin film that we describe as being composed of nodules. Perhaps the terms "protuberance" and "globule" are more appropriate for describing much larger round features that can be seen from the front surface. And in light of the recent reports confirming voids inside thin films $[13,22,35,36]$, "hollow protuberances" may be even more accurate.

Ghosh and Hoek [27] showed that the properties of porous polysulfone supports-particularly pore structure and hydrophobicity — affect the characteristics of the polyamide thin films that are formed on their surfaces by influencing the way in which the amine aqueous solution spreads and fills the support. Here, we were interested in checking whether the polysulfone surface morphology would serve as a mold determining the shape of the polyamide nodules and the overall granular texture observed on the back surfaces of these thin films. Because it was not possible to recover thin films without destroying the underlying support, we looked for indirect evidence by examining the polysulfone supports used by the manufacturers of these two BWRO membranes. According to the AFM results included in Table 1 and the electron micrographs shown in Fig. S2 of the Supplementary Information, both supports are extremely smooth with essentially the same average $R_{R M S}$ value of $6 \mathrm{~nm}$. The support surfaces consist of polysulfone nodules that are not perfectly packed, allowing small pore openings to form between them. 
The minuscule black circles in Fig. S2a and S2c correspond to pore openings with diameters of about 10 $\mathrm{nm}$ and $15 \mathrm{~nm}$ for the polysulfone supports of ESPA3 and AK, respectively. These extremely small pore openings lead to an internal network that increases in pore size along the polysulfone cross-section making the support an effective ultrafiltration membrane but incapable of rejecting dissolved ions. In addition to the roughness difference between the supports and the back surfaces of the polyamide thin films, the sizes of the polysulfone nodules and pores are smaller than the features identified on the back surfaces of the fully aromatic polyamide thin films. Based on the collected microscopic evidence, the back surface of the thin film is not a direct imprint of the polysulfone surface.

The presence of pores is the only other characteristic of back surfaces of MPD-TMC polyamide thin films that has been described [9-13]. We also observed pore openings routinely in our samples (Fig. 2 and Fig. 3S in the Supplementary Information). Their origin is likely an inherent outcome of the MPDTMC IP — not an imprint of the support or a sample preparation artifact-because they were also present in free-standing thin films $[9,12]$. Interestingly, pore sizes match closely the dimensions of the polyamide nodules. Two relatively large pores are highlighted in red in Fig. 2, and their diameters are consistent with the results of Yan et al. who reported a 10-30 nm size range for most pores [13]. However, we did not observe surface porosities as high as the ones detailed in [13]. Pores of these sizes cannot fully cross the thickness of the thin film or else it would be impossible for the thin film to reject ions and other dissolved molecules. Consequently, these openings must lead to cavities that are eventually sealed by polyamide. Yan et al. showed that such pores could extend all the way into the protuberances of the ridge and valley structure [13].

\subsection{TEM tomography of polyamide thin films}

Fig. 3a shows a 2D projection image of the polyamide thin film of ESPA3. As explained in previous work [32], such an image consists of a 2D projection of the thin film volume, which is obtained by placing isolated polyamide thin films perpendicular to the electron beam in the TEM. The dark feature at the center of Fig. 3a corresponds to one of the protuberances (i.e., "ridges"). But in contrast to SEM images like Fig. 1a, Fig. 3a also carries morphological information about the region of the thin film that is underneath the exterior surface of the protuberance. Therefore, it was possible to exploit this advantage with TEM tomography and build accurate 3D visualizations using series of $2 \mathrm{D}$ projection images taken at different tilt angles. Fig. $3 \mathrm{~b}$ shows the area of Fig. 3a tilted to $-70^{\circ}$ (measured from a 
vertical axis through the middle of the image plane). In Fig. 3b, the protuberance remained near the center of the image, but now our vantage point shows its left side compared to Fig. $3 \mathrm{a} .15 \mathrm{~nm}$ gold nanoparticles — appearing clearly as black dots in Fig. 3a-b-were used as fiducial markers (i.e., reference points) to align the images in a stack.
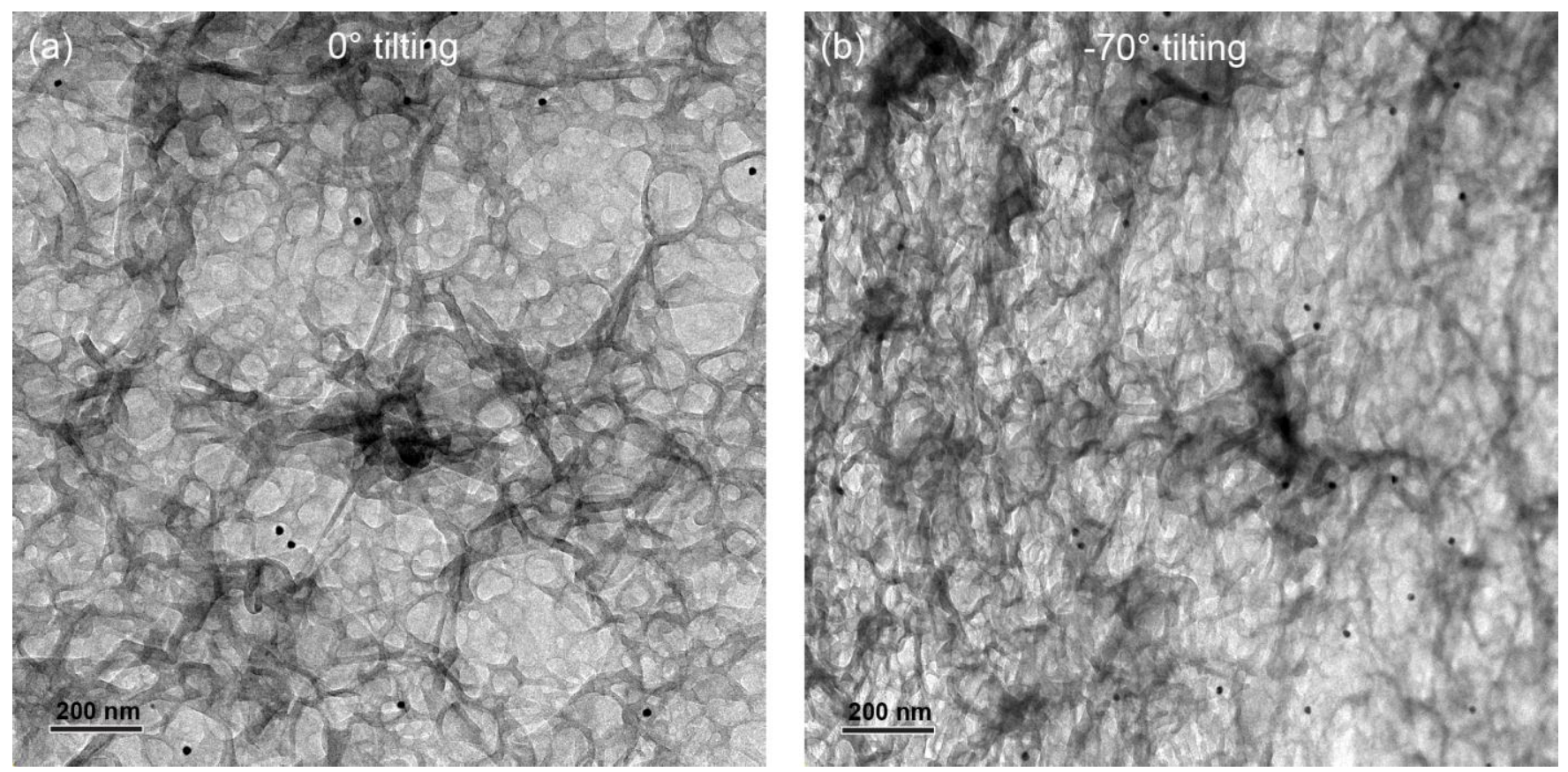

Fig. 3. 2D projection images of the same area of ESPA3 polyamide thin film at (a) $0^{\circ}$ tilting and (b) $-70^{\circ}$ tilting. Black dots are the $15 \mathrm{~nm}$ gold nanoparticles that were used as fiducial markers for constructing the TEM tomogram. Scale bars: $200 \mathrm{~nm}$.

Fig. 4 is an animation of the properly aligned stack moving through the range of tilt angles $\left(-70^{\circ}\right.$ to $+70^{\circ}$ ). The 3D structure of the polyamide thin film is clearly revealed in the animation. The gray areas near the corners appear after the alignment process because the same amount of information is not captured at every tilt angle (e.g., more thin film volume is visible at higher angles). Furthermore, the microscope's limitation to a $140^{\circ}$ tilt range as well as minor shifts of the membrane sample during the acquisition process led to some additional information loss preventing the images from stacking perfectly on top of each other and forcing some orientation corrections during alignment. Nevertheless, based on the smallest discernable details, the resolution of the obtained tomograms was $5 \mathrm{~nm}$ or less in 
the xy-plane, and slightly lower $(\sim 10 \mathrm{~nm})$ in the z-direction (i.e., xz- and yz-cross-sectional views) due to information loss caused by not being able to reach a complete $180^{\circ}$ tilt angle range.

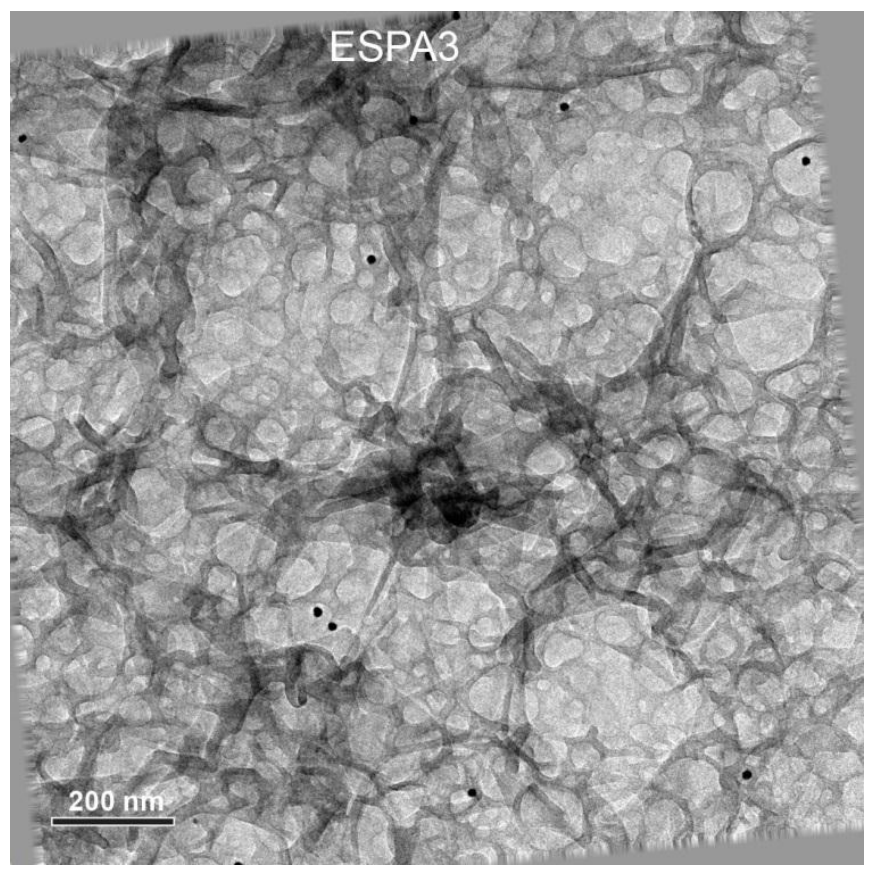

Fig. 4. Video showing the aligned tilt series of 2D projection images as it goes through the entire range of tilt angles $\left(-70^{\circ}\right.$ to $\left.+70^{\circ}\right)$ emphasizing the $3 \mathrm{D}$ structure of the polyamide thin film.

Tomograms were constructed from the aligned image stacks, allowing visualization of any location within the thin film volume. Fig. 5 is a video showing an example of how the tomogram of the polyamide thin film of ESPA3 was explored using virtual slices. In the video, an xy-orthogonal plane (red borders) is shown moving along the thickness of the thin film (z-axis) in a roundtrip starting at the outer most protuberances of the front surface all the way through the film and past the back surface. Two simultaneous vantage points are given: (1) the top view is from a point normal to the xy-plane; (2) the bottom view provides a 3D perspective of the volume and a sense of the relative position of the xyplane with respect to the film thickness. In the bottom view, virtual cross-sections of the thin film can also be seen on the xz- and yz- orthogonal planes (orange and blue, respectively), whose positions are fixed in the animation. The thickness of these virtual slices is approximately $2 \mathrm{~nm}$. 
Fig. 5 shows evident changes in thin film morphology along its thickness. Polyamide is darker gray relative to the background (i.e., open space and internal voids), and as expected, more protuberances appear once the xy-orthogonal plane starts to move down through the thin film. The structure of the thin film becomes more complex with many polymer connections developing between the front-surface protuberances. However, the most striking result is the prevalence of voids throughout the thin film. At the core of the thin film ( $23 \mathrm{~s}$ in the video), the xy-view consists of thin and relatively irregular rings of polymer enclosing empty space. Further along, these polymer rings decrease in size rapidly until a section of mostly polymer and far fewer voids is reached (at $\sim 25 \mathrm{~s}$ ). This constitutes the back surface of the thin film, where several black dots corresponding to deposited gold nanoparticles are also seen. The appearance of the solid back surface and gold nanoparticles at slightly different times for different areas indicates that the polyamide thin film did not lie perfectly flat on the TEM grid. For instance, the back surface was reached first at the bottom part of the frame.
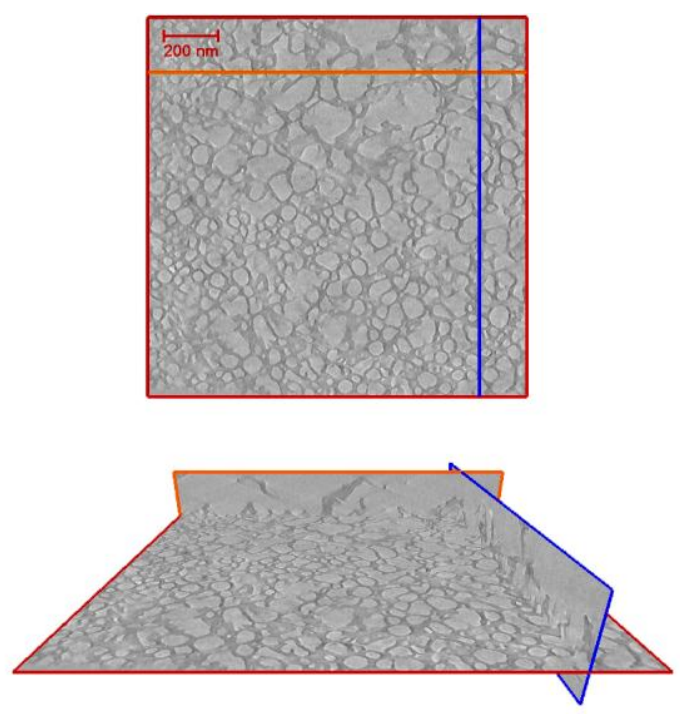

Fig. 5. Video of the tomogram of the ESPA3 polyamide thin film, in which the physical morphology is explored using virtual slices (orthogonal planes). In the video, an xy-orthogonal plane (red) is shown (two simultaneous vantage points) as it travels along the thickness of the thin film (z-axis) from the front to the back surface (and back) revealing how the internal morphology changes at different depths. The orange and blue virtual slices correspond to the xz- and yz- orthogonal planes, respectively. The thickness of these virtual slices is approximately $2 \mathrm{~nm}$. 
In the video, the xy-orthogonal plane goes past the lowest point and then retraces its path back to the front surface. In this second pass, it is possible to observe how the voids within the thin film appear almost immediately at the back surface and then grow in size towards the front surface. An identical video was also prepared for the polyamide thin film of the SWRO membrane SWC3 and is included as Fig. S5 (Supplementary Information). The polyamide thin film of SWC3 shares the same general asymmetric morphology of ESPA3 as seen from the images in Fig. S3 and S4 (Supplementary Information). It is clear from Fig. S5 that the polyamide thin film of SWC3 also contains internal voids. Nonetheless, some significant nanostructural differences were noticed between the two membranes, and their potential implications are discussed in the next section.

\subsection{The internal morphology of fully aromatic polyamide thin films}

Fig. 6 was constructed using virtual slices from the tomograms of the ESPA3 (Fig. 6a-c) and SWC3 (Fig. 6d-f) polyamide thin films. Fig. 6a comprises an xy-orthogonal plane view that is divided vertically in four identical sections, each showing the physical morphology of the thin film at a different position along its thickness (i.e., at different depths in the film). Fig. 6b consists of a yz-orthogonal plane view of the same tomogram, and consequently displays a cross-section of the thin film. The horizontal blue line in Fig. 6a marks the location of the yz-orthogonal plane. Fig. $6 \mathrm{~b}$ is also divided in four sections that match those in Fig. 6a, with the red lines indicating the relative positions of the xy-orthogonal plane with respect to thin-film thickness (z-axis). The position of the xy-orthogonal plane in each section is also expressed as a height $\mathrm{z}$ (in $\mathrm{nm}$ ) using the back surface of the thin film (fourth section) as the reference baseline with $\mathrm{z}=0 \mathrm{~nm}$. Because the polyamide thin film was not perfectly flat, the choice of the baseline was arbitrary considering that a single orthogonal plane did not show a uniform back surface at every point of the frame. Therefore, the baseline does not correspond to the absolute lowest point of the thin film (or the frame), but to a position in which a relatively large fraction of the back surface was visible. As a comparison to Fig. 6a, Fig. $6 \mathrm{c}$ shows the $2 \mathrm{D}$ projection image at $0^{\circ}$ tilting used in the construction of the TEM tomogram. The importance of the tomogram is quite evident as it facilitated the extraction and selective use of the otherwise convoluted and difficult-to-interpret morphological information that is contained in the $2 \mathrm{D}$ projection images. Fig. $6 \mathrm{~d}-\mathrm{f}$ show similar virtual slices for the SWC3 polyamide thin film. Fig. S6 and Fig. S7 in the Supplementary Information show 
the complete image frames (not divided in sections) of the xy-orthogonal plane views at the four different heights z obtained from the ESPA3 and SWC3 tomograms, respectively. 

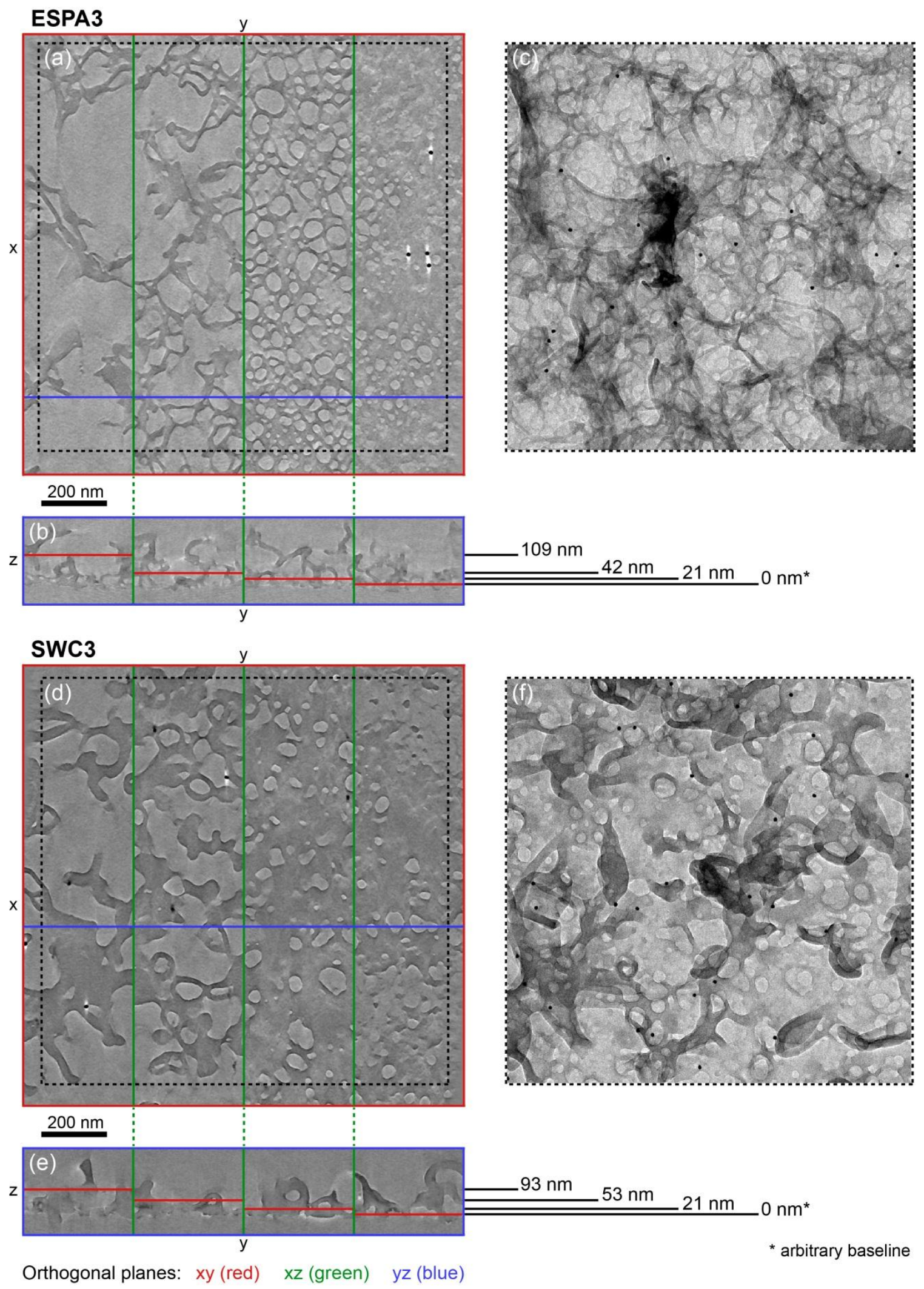
Fig. 6. Orthogonal views extracted from the tomograms of (a-b) ESPA3 and (d-e) SWC3 showing the internal morphology of the polyamide thin films at different heights $\mathrm{z}$, where the baseline $(\mathrm{z}=0 \mathrm{~nm})$ was chosen at a representative location of the back surface. Images (c) and (f) are the 2D projection micrographs at $0^{\circ}$ tilting of ESPA3 and SWC3, respectively, which were used in the creation of the tomograms. The thickness of the virtual orthogonal planes is approximately $2 \mathrm{~nm}$. Scale bars: $200 \mathrm{~nm}$ (identical for all images).

Fig. 6 revealed important structural differences between the internal morphology of the polyamide thin films of ESPA3 and SWC3, despite both being formed by the IP of MPD and TMC. Although voids were also present inside the SWC3 thin film, it is quite evident that there was relatively less empty space (and therefore more polyamide) in the internal nanostructure of SWC3 than in that of ESPA3. The third vertical sections from the left in Fig. 6a and 6d (as well as the corresponding Fig. S6e and S7e in the Supplementary Information) distinctly highlight this difference. These sections show regions deep inside the thin films just above the back surfaces $(z=21 \mathrm{~nm})$ that cannot be observed through any other microscopic technique. At this $\mathrm{z}$, the nanostructure of the ESPA3 thin film consists mostly of voids separated by thin-walled rings of polyamide $(\sim 15 \mathrm{~nm})$, while SWC3 shows fewer voids contained within large areas of solid polyamide. Even when similar polyamide rings appear in the thin film of SWC3 (e.g., at $z=53 \mathrm{~nm}$ ) their walls are clearly thicker than those of ESPA3 (see Fig. 6a, 6d, S6a, S6c, S7a and $\mathrm{S} 7 \mathrm{c})$.

No other characterization technique has established such clear visual differences between the physical morphology of polyamide thin films made with the same monomers, which becomes even more relevant when considering that as a BWRO membrane ESPA3 is significantly more permeable than the SWRO membrane SWC3. Based on the manufacturer's datasheets_-and assuming perfect rejection and no concentration polarization effects — we estimated water permeance values of $6.5 \mathrm{~L} \mathrm{~m}^{-2} \mathrm{~h}^{-1} \mathrm{bar}^{-1}$ and 0.97 $\mathrm{L} \mathrm{m}^{-2} \mathrm{~h}^{-1}$ bar $^{-1}$ for ESPA3 and SWC3, respectively, which are very similar to measured values [43]. ESPA3 is approximately 6 times more permeable than SWC3, and its internal thin film nanostructure qualitatively supports this: thinner polyamide sections and more voids are consistent with lower mass transfer resistance. Yan et al. proposed a conceptual model for the morphology of the polyamide thin film in which every protuberance on the front surface is hollow and connected to at least one of the pore openings on the back surface [13]. Hence, all internal voids are open to the back and the selective barrier 
of the membrane is reduced to single sections of polyamide of about $20 \mathrm{~nm}$ in thickness, which could comprise either the walls of the front-surface protuberances or the thin film base at flat areas [13]. Similar thin-film structures consisting of narrow sections of polyamide $(8-30 \mathrm{~nm})$ and large voids were also reported by others $[21,22,35]$. However, no detailed description was provided regarding how these structural components might be distributed and interconnected within the thin film.

At this stage, it was not possible to obtain comprehensive spatial maps of the internal voids from the tomograms because some locations did not show sufficient contrast between the polyamide and the empty space to accurately reconstruct the complete thin film. Nevertheless, we were able to characterize selected voids from a smaller volume of the ESPA3 thin film, and the results are presented in Fig. 7. Shown in color are 55 representative internal voids of different sizes, whose common characteristic is that they are completely encapsulated as "nanobubbles" within the polyamide thin film. None of these voids is connected to the outside on either side of the thin film, and therefore not accounted for in the conceptual model by Yan et al. [13]. Undoubtedly, some of the pore openings observed on the back surface of the thin film can extend all the way to the front-surface protuberances as discussed in [13], but our results indicate that the thin film also contains voids that are completely enclosed by polyamide.

Each of the nanobubbles in Fig. 7 was created individually by manually delineating its contour in every $2 \mathrm{~nm} x y$-orthogonal plane in which the void appeared along the $\mathrm{z}$-axis. These contours were then combined to generate the void volumes shown. By following each nanobubble along the z-axis (one virtual slice at a time), it was then possible to verify that they were fully enclosed by polyamide. Fig. S8 (Supplementary Information) shows an example of a series of xy-orthogonal planes used for identifying two of the nanobubbles in the ESPA3 thin film. Similar series of xy-orthogonal plane are also included in Fig. S9 and S10 to compare two types of voids in the thin film of SWC3: an encapsulated nanobubble (Fig. S9) and a void open to the back surface (Fig. S10). 
Distribution and shape of selected internal nanobubbles in ESPA3 polyamide thin film

(a) $\mathrm{z}=0 \mathrm{~nm}$
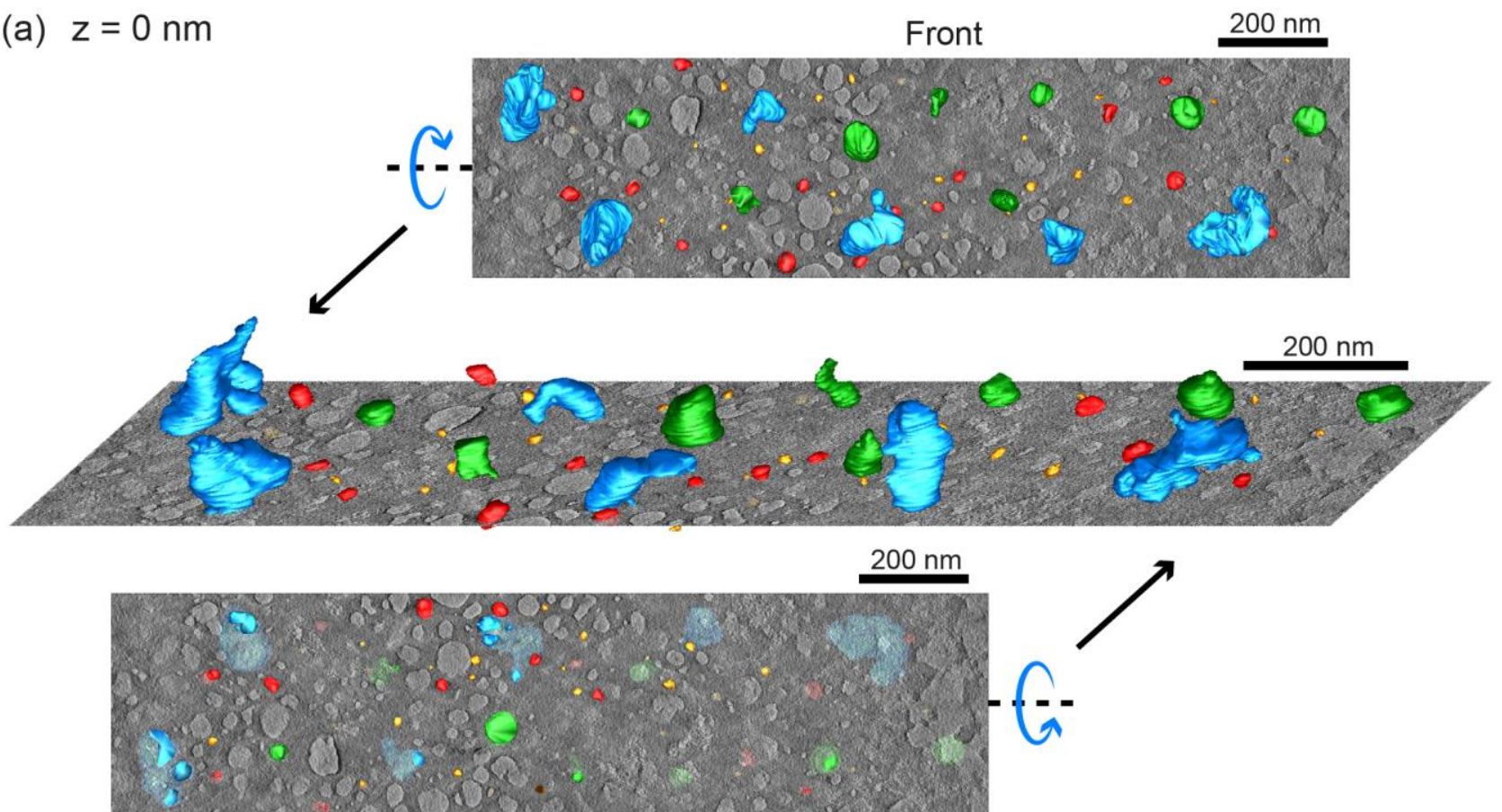

Back

(b) $z=42 \mathrm{~nm}$
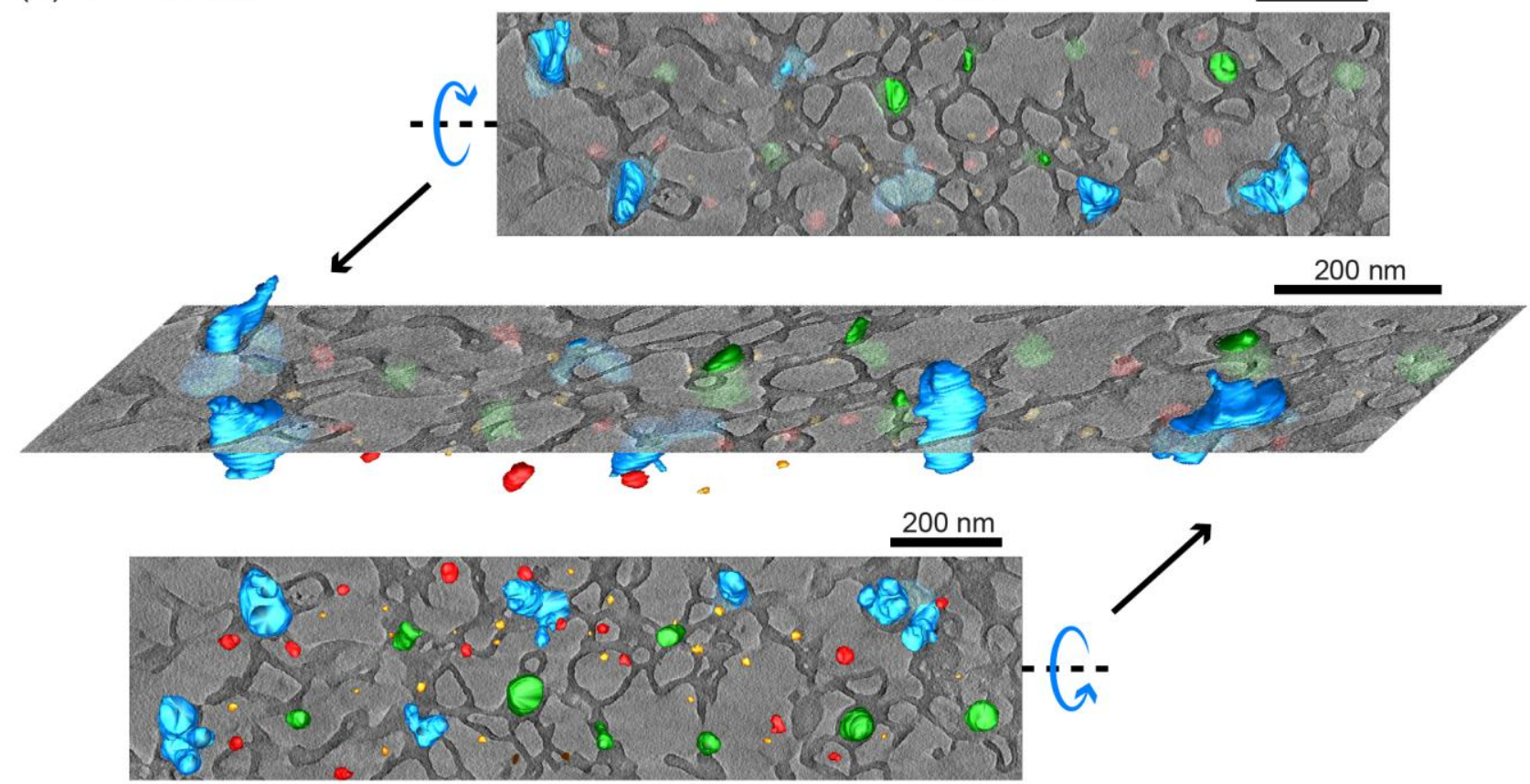

\section{Back}

Void volumes:

$\leq 15$ nm-diameter sphere

$\leq 30 \mathrm{~nm}$-diameter sphere

Single void $>30 \mathrm{~nm}$-diameter sphere

Multiple interconnected voids $>30 \mathrm{~nm}$-diameter sphere 
Fig. 7. 3D visualization of the spatial distribution of nanobubbles of various sizes within the interior of the ESPA3 polyamide thin film. The xy-orthogonal plane is shown at (a) $\mathrm{z}=0 \mathrm{~nm}$ and (b) $\mathrm{z}=42 \mathrm{~nm}$. The top and bottom images in each set correspond to the views from the front and back of the $x y-$ orthogonal plane, respectively. Scale bars: $200 \mathrm{~nm}$.

The center image in Fig. 7a shows the 3D distribution of these voids, as well as the xy-orthogonal plane of the inspected region at $\mathrm{z}=0 \mathrm{~nm}$ (same xy-region shown in the third section of Fig. 6a). The upper and lower images in Fig. 7a provide the corresponding views from above and below the orthogonal plane, respectively. Similarly, Fig. 7b consists of the same arrangement as Fig. 7a except that the xyorthogonal plane is at $\mathrm{z}=42 \mathrm{~nm}$. Image sets at $\mathrm{z}=21 \mathrm{~nm}$ and $\mathrm{z}=109 \mathrm{~nm}$ are included in Fig. S11 of the Supplementary Information. Fig. 7 shows that most voids appear within the first $20 \mathrm{~nm}$ from the back surface and remain extremely small as illustrated by the yellow and red nanobubbles, whose volumes are smaller than those of $15 \mathrm{~nm}$ - and $30 \mathrm{~nm}$-diameter spheres, respectively. Furthermore, the presence of voids so close to the back surface indicates that the segment that we have previously described as the nodular polyamide base is also relatively porous. Other voids grow larger towards the front surface (green nanobubbles) or merge with neighboring ones to form substantial voids (blue nanobubbles) that extend all the way to the front-surface protuberances (see also Fig. S11). Interestingly, we did not observe any internal voids that were be open to the outside on the front-surface side.

The tomogram shows that the nanostructure of the thin film is a continuum of polyamide with an asymmetric distribution of voids increasing in size from the back surface towards the front surface. This is consistent with the postulate that MPD-TMC polyamide thin films grow towards the organic phase [4,9]. The formation of bubble-like voids with equivalent asymmetric size distributions has been documented for other IP processes producing polyamide [44] and polyester [45] films that are, however, orders of magnitude thicker than MPD-TMC thin films. Nonetheless, the film characterized in [44] showed properties typical of polyamide films such as a relatively smoother surface on the aqueous side. For these cases, voids were assumed to form because of aqueous drops appearing on the growing front of the incipient polymer film, thus pushing and reestablishing the organic-aqueous interface at the edges of these drops. As the film grew, retarding monomer diffusion to the interface, aqueous drops had more time to coalesce into bigger drops that ultimately turned into larger internal voids. 


\section{Conclusions}

TEM tomography significantly improved the ability to examine the morphology of polyamide thin films. The resulting tomograms confirmed the existence of substantial empty space in the interior of the thin films and revealed key structural differences between the thin films of ESPA3 (BWRO) and SWC3 (SWRO) that are consistent with their 6-fold difference in water permeance: although both membranes have MPD-TMC polyamide thin films, SWC3 contains fewer voids and thicker polyamide sections than ESPA3. The observed spatial and size distribution of voids is consistent with film growth towards the organic phase during the IP process as most voids originate at the base of the thin film near the back surface and some extend towards the front surface shaping the familiar protuberances of the rough ridge and valley structure. Some voids connect to the outside of the thin film through the pore openings found at the back surface, but others actually comprise nanobubbles completely encapsulated by polyamide. No evidence was found of internal voids opening to the outside on the front-surface side of the thin film. During filtration, these voids are filled with water, and the nanostructural distribution of polyamide and empty space most likely determines the pathways for flow and diffusion across the thin film. . If a pathway goes through one of the nanobubbles then water molecules need to cross at least two sections of polyamide, but water molecules traveling a path through a void open to the back surface cross only one polyamide section. Membrane permeance presumably depends on a combination of pathways that vary in number and thickness of polyamide sections crossed. Therefore, a comprehensive knowledge of the internal morphology of polyamide thin films is essential to better understand mass transport and rejection mechanism in $\mathrm{RO}$ membranes. 


\section{Acknowledgements and note}

Financial support for this work was provided in part by King Abdullah University of Science and Technology (KAUST), the Singapore Stanford Partnership (SSP) and the STC WaterCAMPWS of the National Science Foundation under agreement \#CTS-0120978. Membrane samples were kindly donated by Dr. Craig Bartels of Hydranautics (ESPA3, SWC3) and GE (AK). The authors gratefully acknowledge John Perrino of the Cell Sciences Imaging Facility at Stanford University for his help with the TEM cross-sections and Dr. Eric Litwiller of the KAUST Advanced Membranes and Porous Materials Center (AMPM) for his insightful comments during the preparation of this manuscript. The results herein were first presented at the $8^{\text {th }}$ International Membrane Science and Technology Conference (IMSTEC) in Melbourne, Australia on November $26^{\text {th }}, 2013$. 


\section{References}

[1] J.E Cadotte, R.J. Petersen, Thin-film-composite reverse osmosis membranes: origin, development, and recent advances, in: A.F. Turbak (Ed.), Synthetic Membranes: Vol. I Desalination, ACS Symposium Series 153, American Chemical Society, Washington, D.C., 1981, pp. 305-326.

[2] J.E. Cadotte, Evolution of composite reverse osmosis membranes, in: D.R. Lloyd (Ed.), Materials science of synthetic membranes, ACS Symposium Series 269, American Chemical Society, Washington, D.C., 1985, pp. 273-294.

[3] R.J. Petersen, J.E. Cadotte, Thin film composite reverse osmosis membranes, in: M.C. Porter (Ed.), Handbook of Industrial Membrane Technology, Noyes Publications, New Jersey, 1990, pp. 307-348.

[4] P.W. Morgan, S.L. Kwolek, Interfacial polycondensation. II. Fundamentals of polymer formation at liquid interfaces, J. Polym. Sci. 40 (1959) 299-327.

[5] D.G. Cahill, V. Freger, S.-Y. Kwak, Microscopy and microanalysis of reverse osmosis and nanofiltration membranes, MRS Bull. 33 (2008) 27-32.

[6] S.-Y. Kwak, S.G. Jung, Y.S. Yoon, D.W. Ihm, Details of surface features in aromatic polyamide reverse osmosis membranes characterized by scanning electron and atomic force microscopy, J. Polym. Sci. Pol. Phys. 37 (1999) 1429-1440.

[7] P.M. Johnson, J. Yoon, J.Y. Kelly, J.A. Howarter, C.M. Stafford, Molecular layer-by-layer deposition of highly crosslinked polyamide films, J. Polym. Sci., Part B: Polym. Phys. 50 (2012) 168173.

[8] S. Karan, Z. Jiang, A. Livingston, Sub-10 nm polyamide nanofilms with ultrafast solvent transport for molecular separation, Science 348 (2015) 1347-1351. 
[9] G.-Y. Chai, W.B. Krantz, Formation and characterization of polyamide membranes via interfacial polymerization, J. Membr. Sci. 93 (1994) 175-192.

[10] Y. Jin, Z. Su, Effects of polymerization conditions on hydrophilic groups in aromatic polyamide thin films, J. Membr. Sci. 330 (2009) 175-179.

[11] Q. Li, X. Pan, C. Hou, Y. Jin, H. Dai, H. Wang, Z. Zhao, X. Liu, Exploring the dependence of bulk properties on surface chemistries and microstructures of commercially composite RO membranes by novel characterization approaches, Desalination 292 (2012) 9-18.

[12] J. Lee, A. J. Hill, S. E. Kentish, Formation of a thick aromatic polyamide membrane by interfacial polymerisation. Sep. Purif. Technol. 104 (2013) 276-283.

[13] H. Yan, X. Miao, J. Xu, G. Pan, Y. Zhang, Y. Shi, The porous structure of the fully-aromatic polyamide film in reverse osmosis membranes, J. Membr. Sci. 475 (2015) 504-510.

[14] C.Y. Tang, Y.-N. Kwon, J.O. Leckie, Effect of membrane chemistry and coating layer on physiochemical properties of thin film composite polyamide RO and NF membranes I. FTIR and XPS characterization of polyamide and coating layer chemistry, Desalination 242 (2009) 149-167.

[15] C.Y. Tang, Y.-N. Kwon, J.O. Leckie, Effect of membrane chemistry and coating layer on physiochemical properties of thin film composite polyamide RO and NF membranes II. Membrane physiochemical properties and their dependence on polyamide and coating layers, Desalination 242 (2009) 168-182.

[16] C.Y. Tang, Y.-N. Kwon, J.O. Leckie, Probing the nano- and micro-scales of reverse osmosis membranes-A comprehensive characterization of physiochemical properties of uncoated and coated membranes by XPS, TEM, ATR-FTIR, and streaming potential measurements, J. Membr. Sci. 287 (2007) 146-156. 
[17] P. Marchetti, M.F. Jimenez Solomon, G. Szekely, A.G. Livingston, Molecular separation with organic solvent nanofiltration: A critical review, Chem. Rev. 114 (2014) 10735-10806.

[18] A.K. Ghosh, B.-H. Jeong, X. Huang, E.M.V. Hoek, Impacts of reaction and curing conditions on polyamide composite reverse osmosis membrane properties, J. Membr. Sci. 311 (2008) 34-45.

[19] M. Hirose, H. Ito, Y. Kamiyama, Effect of skin layer surface structures on the flux behaviour of RO membranes, J. Membr. Sci. 121 (1996) 209-215.

[20] S.-Y. Kwak, S.G. Jung, S.H. Kim, Structure-motion-performance relationship of flux-enhanced reverse osmosis (RO) membranes composed of aromatic polyamide thin films, Environ. Sci. Technol. 35 (2001) 4334-4340.

[21] C. Kong, M. Kanezashi, T. Yamomoto, T. Shintani, T. Tsuru, Controlled synthesis of high performance polyamide membrane with dense layer for water desalination, J. Membr. Sci. 362 (2010) 76-80.

[22] T. Kamada, T. Ohara, T. Shintani, T. Tsuru, Optimizing the preparation of multi-layered polyamide membrane via the addition of a co-solvent, J. Membr. Sci. 453 (2014) 489-497.

[23] S.-Y. Kwak, D.W. Ihm, Use of atomic force microscopy and solid-state NMR spectroscopy to characterize structure-property-performance correlation in high-flux reverse osmosis (RO) membranes, J. Membr. Sci. 158 (1999) 143-153.

[24] S. Hermans, R. Bernstein, A. Volodin, I.F.J. Vankelecom, Study of synthesis parameters and active layer morphology of interfacially polymerized polyamide-polysulfone membranes, React. Funct. Polym. 86 (2015) 199-208.

[25] I.-C. Kim, B.-R. Jeong, S.-J. Kim, K.-H. Lee, Preparation of high flux thin film composite polyamide membrane: The effect of alkyl phosphate additives during interfacial polymerization, Desalination 308 (2013) 111-114. 
[26] P.S. Singh, S.V. Joshi, J.J. Trivedi, C.V. Devmurari, A. Prakash Rao, P.K. Ghosh, Probing the structural variations of thin film composite RO membranes obtained by coating polyamide over polysulfone membranes of different pore dimensions, J. Membr. Sci. 278 (2006) 19-25.

[27] A.K. Ghosh, E.M.V. Hoek, Impacts of support membrane structure and chemistry on polyamidepolysulfone interfacial composite membranes, J. Membr. Sci. 336 (2009) 140-148.

[28] M.F. Jimenez-Solomon, P. Gorgojo, M. Munoz-Ibanez, A.G. Livingston, Beneath the Surface: Influence of supports on thin film composite membranes by interfacial polymerization for organic solvent nanofiltration, J. Membr. Sci. 448 (2013) 102-113.

[29] V. Freger, Nanoscale heterogeneity of polyamide membranes formed by interfacial polymerization, Langmuir 19 (2003) 4791-4797.

[30] C.Y. Tang, Y.-N. Kwon, J.O. Leckie, Characterization of humic acid fouled reverse osmosis and nanofiltration membranes by transmission electron microscopy and streaming potential measurements, Environ. Sci. Technol. 41 (2007) 942-949.

[31] G.E. Mitchell, B. Mickols, D. Hernandez-Cruz, A. Hitchcock, Unexpected new phase detected in FT30 type reverse osmosis membranes using scanning transmission X-ray microscopy, Polymer 52 (2011) 3956-3962.

[32] F.A. Pacheco, I. Pinnau, M. Reinhard, J.O. Leckie, Characterization of isolated polyamide thin films of RO and NF membranes using novel TEM techniques, J. Membr. Sci. 358 (2010) 51-59.

[33] F.A. Pacheco Oreamuno, Microscopic characterization of the nanostructure of polyamide thin films in reverse osmosis and nanofiltration membranes, Ph.D. dissertation, Stanford University, Stanford, CA, 2011. 
[34] C. Kong, A. Koushima, T. Kamada, T. Shintani, M. Kanezashi, T. Yoshioka, T. Tsuru, Enahanced performance of inorganic-polyamide nanocomposite membranes prepared by metal-alkoxide-assisted interfacial polymerization, J. Membr. Sci. 366 (2011) 382-388.

[35] T. Tsuru, S. Sasaki, T. Kamada, T. Shintani, T. Ohara, H. Nagasawa, K. Nishida, M. Kanezashi, T. Yoshioka, Multilayered polyamide membranes by spray-assisted 2-step interfacial polymerization for increased performance of trimesoyl chloride (TMC)/m-phenylenediamine (MPD)-derived polyamide membranes, J. Membr. Sci. 446 (2013) 504-512.

[36] M. Kurihara, M. Hanakawa, Mega-ton water system: Japanese national research and development project on seawater desalination and wastewater reclamation, Desalination 308 (2013) 131-137.

[37] J.H. Moon, A.R. Katha, S. Pandian, S.M. Kolake, S. Han, Polyamide-POSS hybrid membranes for seawater desalination: Effect of POSS inclusion on membrane properties, J. Membr. Sci. 461 (2014) 8995 .

[38] J. Duan, Y. Pan, F. Pacheco, E. Litwiller, Z. Lai, I. Pinnau, High-performance polyamide thin-filmnanocomposite reverse osmosis membranes containing hydrophobic zeolitic imidazolate framework-8, J. Membr. Sci. 476 (2015) 303-310.

[39] F.H. She, K. Nihara, W.M. Gao, P.D. Hodgson, H. Jinnai, L.X. Kong, 3-Dimensional characterization of membrane with nanoporous structure using TEM tomography and image analysis, Desalination 250 (2010) 757-761.

[40] S.P. Nunes, A.R. Behzad, B. Hooghan, R. Sougrat, M. Karunakaran, N. Pradeep, U. Vainio, K.-V. Peinemann, Switchable $\mathrm{pH}$-responsive polymeric membranes prepared via block copolymer micelle assembly, ACS Nano 5 (2011) 3516-3522.

[41] W.O. Saxton, W. Baumeister, M. Hahn, Three-dimensional reconstruction of imperfect twodimensional crystals, Ultramicroscopy 13 (1984) 57-70. 
[42] S. Bason, Y. Oren, V. Freger, Ion transport in the polyamide layer of RO membranes: Composite membranes and free-standing films, J. Membr. Sci. 367 (2011) 119-126.

[43] J.S. Louie, I. Pinnau, M. Reinhard, Gas and liquid permeation properties of modified interfacial composite reverse osmosis membranes, J. Membr. Sci. 325 (2008) 793-800.

[44] L.J.J.M. Janssen, K. te Nijenhuis, Encapsulation by interfacial polycondensation. I. The capsule production and a model for wall growth, J. Membr. Sci. 65 (1992) 59-68.

[45] F. Yuan, Z. Wang, X. Yu, Z. Wei, S. Li, J. Wang, S. Wang, Visualization of the formation of interfacially polymerized film by an optical contact angle measuring device, J. Phys. Chem. C 116 (2012) 11496-11506. 


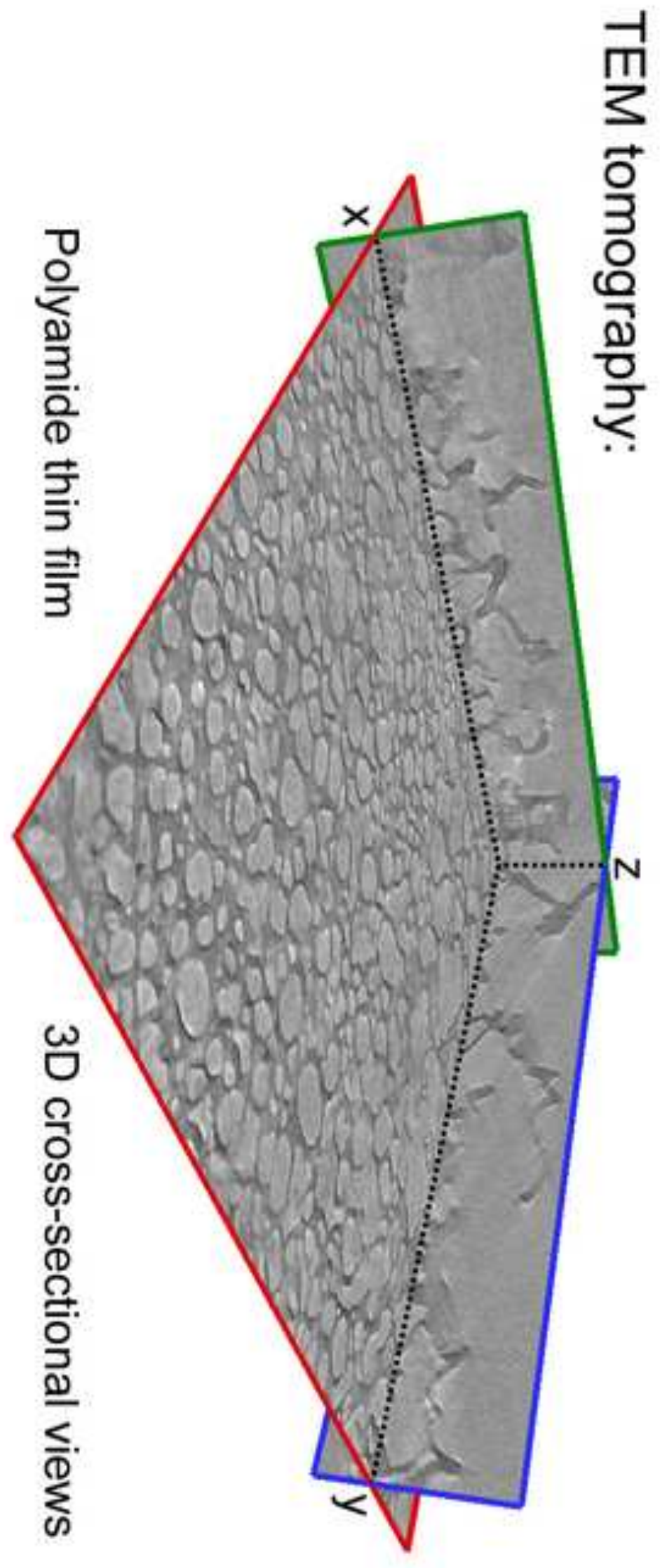

